\title{
ANÁLISIS EN LA RELACIÓN ENTRE EL FONDO \\ DE MANIOBRA Y LA RENTABILIDAD: \\ CASO DEL SECTOR OLEÍCOLA ESPAÑOL
}

\author{
ALBERTO LÓPEZ PÉREZ \\ Universidad de San Carlos de Compostela, España \\ albertolp1188@gmail.com \\ SARA FERNÁNDEZ LÓPEZ \\ Universidad de San Carlos de Compostela, España \\ sara.fernadez.lopez@usc.es \\ DAVID RODEIRO PAZOS \\ Universidad de San Carlos de Compostela, España \\ david.rodeiro@usc.es \\ FEDERICO LI BONILLA \\ Universidad Estatal a distancia, Costa Rica \\ fli@uned.ac.cr
}

\section{RESUMEN}

La estacionalidad de la actividad oleica, incide que los flujos de caja por ventas de contado y a crédito. Sus plazos y recuperación de efectivo, afectan el rendimiento financiero de las empresas involucradas en dicha actividad. En el entorno empresarial se rumora que dicha actividad oleica es de baja rentabilidad, lo cual motiva esta investigación, para poder anticipar o corregir la gestión del capital de maniobra y mejorar la rentabilidad. Para llevar a cabo esta pesquisa, se utilizó una base de datos con 817 empresas del sector. Una vez depurada la muestra, esta quedó en 324 empresas para realizar el análisis entre la relación de fondo de maniobra y la rentabilidad y poder determinar si el fondo de maniobra o capital de trabajo incide en la baja rentabilidad o no. El valor del aporte de esta investigación, a la academia y al mundo empresarial, es sobre la importancia del fondo de maniobra y su rentabilidad en la gestión de Pymes; en especial para las empresas que se dedican a la extracción, manipulación y envasado a granel así como al detal del aceite de oliva. Para ello, los análisis realizados rechazan las cuatro hipótesis de este trabajo. La contribución de esta investigación se da en que, en los hallazgos obtenidos, existe una relación positiva entre la gestión del fondo de maniobra y la rentabilidad. Se da una diferencia de rentabilidad entre cooperativas y no cooperativas. Estos hallazgos sugieren que las bajas tasas de interés en España y la flexibilidad en el plazo venta del aceite y de su cobro, no afectan su rentabilidad en forma negativa.

PALABRAS CLAVE: FONDO DE MANIOBRA, RENTABILIDAD, AGROINDUSTRIA OLEICA, GESTIÓN, PYMES, COOPERATIVAS.

\section{ABSTRACT}

The seasonality of the oleic activity, impacts the cash flows from cash and credit sales, their terms and cash recovery, affecting the financial performance of the companies involved in this activity. In the business environment it is rumored that said oleic 
activity is of low profitability; which motivates this investigation, in order to be able to anticipate or correct the management of working capital and improve profitability. To carry out this research, a database was used with 817 companies in the sector. Once the sample was cleaned, it was left in 324 companies to carry out the analysis of the relation between the rolling fund and profitability, and be able to determine if the rolling fund or working capital affects the low profitability or not. The value of the contribution of this research to the academy and the business world is on the importance of working capital and its profitability in the management of SMEs, especially for companies engaged in the extraction, handling and packaging in bulk as well as retail of olive oil. For which the analysis carried out reject the four hypotheses of this work. The contribution of this research is given in that the findings reveal there is a positive relationship between the management of the working capital and profitability. There is a difference in profitability between cooperatives and non-cooperatives. Given that our findings suggest that the low interest rates in Spain and the flexibility in the sale time of the oil and its collection, does not affect its profitability negatively.

KEYWORDS:WORKINGCAPITAL,PROFITABILITY, OLEIC AGROINDUSTRY, MANAGEMENT, SMES, COOPERATIVES.

\section{INTRODUCCIÓN}

Las finanzas corporativas han centrado su atención en el estudio de las decisiones financieras a largo plazo (Baños-Caballero, García-Teruel y Martinez-Solano, 2014; Nazir y Afza, 2009), relegando a un segundo plano la financiación a corto plazo, cuando esta es la principal forma de financiación de las pequeñas y medianas empresas (Pymes) (Enow y Brijlal, 2014). Se relegan, asimismo, otras decisiones financieras que afectan a la gestión de los activos y pasivos corrientes o, en definitiva, del fondo de maniobra.
En este sentido, Howorth y Westhead (citados en Serrasqueiro, 2014) sostienen que la gestión del circulante es más importante para las Pymes, pues en estas tiene mayor repercusión sobre la rentabilidad que en las empresas de mayor tamaño, al tener mayor porcentaje de activos corrientes, menor liquidez, flujos de caja más volátiles y mayor dependencia sobre la deuda a corto plazo. Nazir y Afza (2009) comparten dicha opinión, advirtiendo que el éxito del negocio depende en gran medida de la habilidad de los directores financieros a la hora de gestionar las existencias, los deudores y los acreedores.

Por este motivo, las empresas deberán destinar importantes recursos al mantenimiento de un adecuado fondo de maniobra. De hecho, la ausencia de fondo de maniobra es una de las principales causas del fracaso de los negocios de menor tamaño (Padachi, 2006). En concordancia con lo anterior, otros autores defienden que, cuando surgen necesidades financieras, es mejor recurrir a financiación a largo plazo que modificar las políticas de gestión de circulante, si esto fuera posible (Serrasqueiro, 2015).

Debido al gran peso de las Pymes en la economía española y dada la coyuntura económica actual, donde se atraviesa uno de los momentos con mayores restricciones financieras, crece la importancia de la gestión del fondo de maniobra. Dicha gestión resulta aún más relevante cuando se analiza el caso de empresas productoras del sector agrario que, con frecuencia, se caracterizan por una producción de carácter estacional, donde los precios de la materia prima experimentan importantes volatilidades al depender de cuestiones externas al control de las empresas, fundamentalmente avatares climatológicos. A pesar de ello, son relativamente escasos los trabajos que analizan el efecto de la gestión en el fondo de maniobra sobre la rentabilidad empresarial y, en concreto, los que se centran en empresas del sector agrario. 
Este estudio busca cubrir esta brecha de la literatura. El objetivo de este trabajo es analizar si la gestión del fondo de maniobra ejerce algún efecto sobre la rentabilidad económica de las empresas españolas dedicadas a la producción de aceite de oliva. Para ello, se utiliza una muestra de 324 empresas del sector oleícola durante el periodo 2010-2014 y se aplica metodología de datos de panel.

Analizar la gestión y rentabilidad empresarial de este sector resulta crucial por varios motivos. En primer lugar, se trata de un sector agrario clave para la economía española, puesto que su producción supone aproximadamente el 54\% de la producción mundial y el 72\% de la Unión Europea (MAGRAMA, 2016) y genera aproximadamente 8000 puestos de trabajo (ICEX, 2012), constituyendo el producto exportado más significativo del sector agroalimentario (MAGRAMA, 2016). En segundo lugar, las empresas del sector oleícola tienen una rentabilidad muy reducida, que oscilaba entre el $1.6 \%$ y el $4.6 \%$, según datos del 2012 (Analistas económicos de Andalucía, 2012), tratándose, además, de empresas de reducido tamaño y que enfrentan importantes restricciones de crédito (Llano, 2014). Por tanto, ayudar a entender la rentabilidad de las empresas del sector contribuye a garantizar la supervivencia de este.

Para alcanzar dicho objetivo, el resto del trabajo se estructura en cinco apartados. Tras esta introducción, el segundo título revisa los argumentos y resultados de los trabajos empíricos que analizaron los efectos del fondo de maniobra en la rentabilidad de las empresas. En el tercer apartado, se realiza una breve caracterización a las empresas del sector oleícola español, con el objetivo de contextualizar el análisis realizado. En el cuarto título, se detalla la metodología utilizada para, a continuación, realizar el análisis descriptivo y multivariable, para someter a comprobación las hipótesis de estudio. Finalmente, se concluye resumiendo los principales resultados obtenidos para luego dar algunas recomendaciones de cara a la gestión del fondo de maniobra, así como las limitaciones surgidas en la realización de este trabajo y las futuras líneas de investigación.

\section{REVISIÓN DE LA LITERATURA}

El fondo de maniobra, en caso de ser positivo, representa la parte de los activos corrientes que se financian con recursos permanentes; mientras que, si es negativo, representa la parte de los activos no corrientes que son financiados con recursos a corto plazo. De este modo, a mayor inversión en el fondo de maniobra, mayores serán las necesidades de financiación, perjudicando la rentabilidad de la empresa. Ahora bien, disponer de un mayor fondo de maniobra permitirá a las empresas reducir parte de los riesgos a los que están expuestas (Shin y Soenen, 1998). Las empresas que opten por tener un fondo de maniobra elevado estarán llevando a cabo una política de gestión catalogada como conservadora.

Frente a esta política conservadora, las empresas que opten por políticas más agresivas en la gestión del fondo de maniobra, estarán incrementando el riesgo asumido en busca de mayor rentabilidad (Nazir y Afza, 2009; Serrasqueiro, 2015; Vishnani y Shah, 2007). Ahora bien, estas políticas agresivas suelen tener un límite, ya que los costes de tener un fondo de maniobra reducido se incrementarían proporcionalmente más que el incremento de los beneficios (Rezaei y Pourali, 2015). Esto se debería a que la ausencia de liquidez impediría que las empresas pudieran afrontar las obligaciones a corto plazo, además de ocasionar interrupciones en su actividad (Padachi, 2006).

A continuación, se hace una revisión de los trabajos empíricos que recientemente han estudiado la relación entre cada uno de los componentes del fondo de maniobra y la ren- 
tabilidad empresarial (tabla 1) para plantear las hipótesis de estudio.

\section{GESTIÓN DE DEUDORES}

La gestión del cobro a deudores suele representarse a través del plazo de cobro a deudores. Dicho plazo, expresa el número de días que transcurren desde que se vende un producto hasta que se cobra por su venta. Cabe esperar que la gestión eficaz de dicho plazo tenga una repercusión positiva en la rentabilidad de las empresas (Deloof, 2003; García-Teruel y Martínez-Solano, 2007; Jayarathne, 2014; Serrasqueiro, 2015), pues está estrechamente relacionado con las ventas (Jayarathne, 2014).

A la hora de gestionar las políticas de cobro, las empresas pueden optar por permitir a los clientes pagar en un intervalo de días más amplio o más estrecho. Por un lado, ofrecer un mayor plazo repercutirá negativamente en la rentabilidad, al implicar una mayor financiación de los clientes (con su correspondiente coste de oportunidad), además de incrementar el riesgo comercial (riesgo de impago) al crecer el porcentaje de deudas incobrables, como indica Bhattacharya (citado en Jayarathne, 2014).

Por otro lado, el brindar un plazo sea más amplio puede tener un efecto positivo en la rentabilidad. Esto se debe a que mediante el plazo de cobro los clientes de la empresa obtienen financiación a un coste generalmente menor al ofertado por las entidades financieras, ya que la empresa tiene determinadas ventajas sobre aquellas a la hora de conceder crédito y, además, se les está permitiendo probar el producto antes de pagarlo (Deloof, 2003; García-Teruel y Martínez-Solano, 2007; Lazaridis y Tryfonidis, 2006).

Estas facilidades actuarán como valor añadido a la hora de escoger entre alternativas de productos similares (Baños-Caballero et al., 2014), de modo que posibilitará un aumento en las ventas, consolidar relaciones a largo plazo con los clientes e, incluso, atender a un segmento de clientes que solo pueda comprar a crédito, repercutiendo positivamente en la rentabilidad de la empresa. De hecho, Emery opina que ofrecer crédito comercial es una inversión a corto plazo más rentable que el mercado de valores (citado en Deloof, 2003).

Los resultados empíricos no son concluyentes al respecto; la relación entre el plazo de cobro y la rentabilidad de las empresas es negativa (ver tabla 1). Los autores atribuyen este resultado negativo no solo al incremento que se produce en los costes de oportunidad de la empresa, al tener que financiar en mayor medida su capital circulante, sino también a la posibilidad de que los clientes con más problemas de liquidez acaben seleccionando a aquellas empresas que ofrecen periodos de pago más extensos, generando, en el largo plazo, problemas de impagados y pérdidas de rentabilidad. Con base en la literatura previa, se propone la siguiente hipótesis:

H1: Se espera una relación negativa entre el plazo de cobro a deudores y la rentabilidad de la empresa.

\section{GESTIÓN DE INVENTARIOS}

La gestión de inventarios se tiende a representar con el plazo de existencias, esto es, el período de tiempo que comprende desde que se compran las existencias hasta que se venden. Se espera que la correcta gestión de la política de inventarios provoque un incremento en la rentabilidad de las empresas (Deloof, 2003; García-Teruel y Martínez-Solano, 2007; Rezaei y Pourali, 2015).

Mantener un inventario medio (plazo de existencias) mayor tendrá una influencia negativa en la rentabilidad, dado que generará más costes de almacenamiento, seguros, deterioros y mermas, así como un incremento del coste de oportunidad al tener el dinero inmovilizado 
en las partidas anteriores (Serrasqueiro, 2015). Además, en ocasiones, estos mayores plazos pueden ser consecuencia de una caída de las ventas (Deloof, 2003).

Por el contrario, un incremento del plazo de existencias (inventario medio) permitirá a las empresas: disminuir los riesgos de ruptura de stock (Baños-Caballero et al., 2014; Deloof, 2003; Vishnani y Shah, 2007), junto con los inconvenientes inherentes a esta (ventas perdidas, peor reputación, interrupción de los procesos productivos...). También, se posibilita reducir los costes de suministro (García-Teruel y Martínez-Solano, 2007), al poder realizar mayores cantidades de pedido, aprovechando descuentos por cantidad u otras ofertas y, consecuentemente, reducir el número de pedidos y cubrirse ante fluctuaciones de los precios (Baños-Caballero et al., 2014).

La mayoría de los trabajos empíricos muestran una relación negativa entre la rentabilidad de la empresa y su periodo de existencias (Deloof, 2003; Raheman y Nasr, 2007; García-Teruel y Martínez-Solano 2007; Vahid, Elham, Khosroshahi-Mohsen, y Mohammadreza, 2012; Serrasqueiro, 2015; Musau, 2015). Con los resultados anteriores como motivo, a la hora de desarrollar el trabajo empírico se parte de la siguiente hipótesis:

H2: Se espera una relación negativa entre el plazo de existencias y la rentabilidad de la empresa.

\section{GESTIÓN DE PROVEEDORES}

Una óptima gestión de la política de pago a proveedores debería tener un efecto positivo en la rentabilidad de las empresas. El principal aspecto a gestionar será el plazo de pago a proveedores, plazo que plasma el número de días que se difiere el pago de una compra con respecto a la fecha en que se realizó.

A medida que se incrementa este plazo, menores serán las necesidades de financiación bancaria, pues los proveedores estarán financiando a las empresas a un coste inferior al ofrecido por las entidades financieras (García-Teruel y Martínez-Solano, 2007). El hecho de acceder a una financiación más barata implica que se podrán dedicar recursos a otras inversiones y obtener mayor rentabilidad (Serrasqueiro, 2015). Igualmente, se podrá recurrir al límite ocioso de financiación bancaria, inicialmente destinado a costear circulante, para financiar otras inversiones.

Este uso del crédito de proveedores como fuente alternativa de financiación adquiere mayor importancia en países donde los mercados de capitales están menos desarrollados, a causa de la fuerte presencia de los problemas de agencia. En dichos países, el crédito de proveedores puede usarse para financiar el crecimiento de la empresa, mitigando el menor acceso al crédito bancario (Deloof, 2003). Un razonamiento similar tendría lugar en momentos del tiempo en los cuales existe una restricción importante de otros recursos financieros externos.

El aspecto negativo de demorar el pago a proveedores reside en un incremento del coste de oportunidad, al renunciar a descuentos por pronto pago (Deloof, 2003; García-Teruel y Martínez-Solano, 2007)'. En épocas de bajos tipos de interés, como sucede en la actualidad, el descuento por pronto pago ofrecido por lo proveedores suele suponer un coste implícito de financiación muy superior al coste de la financiación bancaria. En ese caso, sería más interesante acceder a los descuentos por pronto pago y renunciar a la financiación de los proveedores, sustituyéndola por financiación bancaria.

1 Dicho esto, Deloof (2003) expresa que los descuentos por pronto pago se contabilizarían como ingresos financieros (según los criterios contables belgas), por lo que no afectaría a la rentabilidad económica. En España, sí tendrían repercusión en la rentabilidad económica, siempre y cuando se incluyeran en la factura, pues disminuirían el importe del consumo de mercaderías y no irían como ingresos financieros. Si en vez de rentabilidad económica se usara la rentabilidad financiera, estos descuentos sí le afectarían, independientemente de si se incluyeran o no en la factura. 
Sorprendentemente, los trabajos empíricos encontraron mayoritariamente una relación negativa entre la rentabilidad de la empresa y su plazo de pago a proveedores (Deloof, 2003; García-Teruel y Martínez-Solano, 2007; Gul, Khan, Raheman, Khan, Khan, y Khan, 2013; Reheman y Nasr, 2007; Serrasqueiro, 2015; Taghizadeh, Ghanavati, Akbari, y Mohammadreza, E., 2012). Algunos autores explican que esta relación se debía a un empeoramiento de las relaciones comerciales con los proveedores, al incrementar sus costes al financiar a la empresa (Jayarathne, 2014). Otra explicación, según Deloof (2003), es que las empresas menos rentables tardan más tiempo en pagar, por lo que tendría lugar una relación causa-efecto inversa. A la hora de desarrollar el trabajo empírico, se parte de la siguiente hipótesis:

H3: Se espera una relación positiva entre el plazo de pago a proveedores y la rentabilidad de la empresa.

\section{CICLO DE TESORERÍA}

El ciclo de tesorería es la medida resultante de restar el plazo de pago a proveedores a la suma del plazo de existencias y el plazo de cobro a deudores. Esta medida representa el número de días que transcurren desde que se paga la compra de existencias hasta que se cobra por su venta. Comúnmente, se usa para representar la eficiencia en la gestión del fondo de maniobra (Gentry, Vaidyanathan, y Lee 1990; Nobanee, 2009; Vural, Sökmen, y Çetenak., 2012).

Los argumentos que relacionan la rentabilidad de la empresa con el ciclo de tesorería son, en resumen, los que están detrás de cada uno de sus componentes: plazos de cobro, pago y existencias. Un incremento del ciclo de tesorería generará un incremento del fondo de maniobra y, por consiguiente, de las necesidades de financiación de este (Vural et al., 2012). Se espera, por lo tanto, una relación negativa entre el ciclo de tesorería y la rentabilidad de las empresas (Nobanee, 2009;
Shin y Soenen, 1998). Por el contrario, cuanto más frecuente sea el ciclo de tesorería, mayores serán las ventas, lo cual puede incrementar la rentabilidad siempre que el coste de la mayor inversión en el fondo de maniobra crezca a un ritmo menor que los beneficios de mantener dicho inventario elevado (Deloof, 2003).

En el pasado, diferentes autores han estudiado empíricamente esta relación y han encontrado una relación negativa entre ambas variables (ver tabla 1). Tomando como base dichos resultados, se establece la siguiente hipótesis:

H4: Se espera una relación negativa entre el ciclo de tesorería y la rentabilidad de la empresa.

\section{EL SECTOR OLEICO ESPAÑOL}

El objetivo de esta sección es introducir las principales características de las empresas que integran el sector oleícola español y cómo pueden influir en sus decisiones de gestión del fondo de maniobra. La fabricación de aceite de oliva es una actividad de gran relevancia en España, país que representa aproximadamente el 54\% de la producción mundial y el 72\% de la Unión Europea. Además, el aceite de oliva constituye el producto exportado "más representativo" del sector agroalimentario (MAGRAMA, 2015). También, es relevante el número de empleos que crea (aproximadamente 8000 según ICEX, 2012).

Las empresas cuya actividad principal es la fabricación de aceite de oliva están recogidas bajo el epígrafe 1043 de la Clasificación Nacional de Actividades Económicas (CNAE). Según AICA (2016), el sector estaba integrado por 1775 almazaras (industria o molino donde se extrae el aceite de oliva) en 2016. Dentro de dicho grupo, el 53\% estaban agrupadas como cooperativas y generaban el $67 \%$ de la producción total de aceite de oliva, mientras que el $47 \%$ restante son almazaras agrícolas e industriales y les correspondía el 33\% de la producción total (AICA, 
2016). Además, la cuota de mercado está muy concentrada, ya que las diez empresas de mayor facturación representan el 85\% de la facturación total (El Economista, 2015).

Se trata de empresas de reducido tamaño; el $80.4 \%$ de las empresas tiene menos de 10 empleados; el $17.6 \%$ entre 10 y 50 empleados y solamente el $2 \%$ tiene más de 50 empleados (Analistas económicos de Andalucía, 2012). Además, las empresas tienen un ratio de dependencia financiera superior al 50\%, por lo que la financiación con recursos ajenos predomina sobre los recursos propios (Llano, 2014). Profundizando en la financiación con recursos ajenos, las grandes empresas están financiadas principalmente con pasivos a corto plazo (Empresa Actual, 2010), lo que repercute en un mayor riesgo a corto plazo.

El sector oleícola tiene una rentabilidad muy reducida. Una parte de las almazaras se dedica a envasar el aceite que producen, mientras que otras se dedican a venderlo a granel a las empresas envasadoras. La diferencia entre envasar lo producido o vender el aceite a granel tiene gran repercusión en la rentabilidad, siendo la rentabilidad económica de los primeros del 4.6\% aproximadamente, frente al 1.6\% que ganan aproximadamente los segundos (Analistas económicos de Andalucía, 2012), según datos del 2008. Dicha rentabilidad reducida, unida a un esperado incremento de los costes de aprovisionamiento (CESCE, 2015), motiva que resulte de gran interés estudiar cómo afecta la gestión del fondo de maniobra a la misma.

Además, la elevada volatilidad de los precios, derivada de la propia volatilidad de la producción de aceite, podría provocar una reducción de los márgenes de explotación y pondría en riesgo a las explotaciones menos rentables (Analistas económicos de Andalucía, 2013). Para tratar de mitigar este fenómeno, surgen las ayudas al almacenamiento privado, consis- tentes en la concesión de subvenciones públicas al almacenamiento durante un período determinado, normalmente 180 días (FEGA, 2009), para que los productores no liquiden sus existencias de forma inmediata ante potenciales restricciones de crédito². Esta medida fue empleada durante los años 2011 y 2012 (FEGA, 2017), que forman parte del horizonte de estudio considerado.

Finalmente, destaca la venta a granel en grandes cantidades (Analistas económicos de Andalucía, 2012). En este caso, el poder de negociación del comprador será mayor que el del vendedor, debido a que los compradores están más concentrados (Briega, 2014), lo que desembocará en facilidades de pago como es la financiación de las compras.

\section{METODOLOGÍA}

Es una investigación cuantitativa y transversal, para la cual se utiliza la base de datos SABI de Bureau Van Dijk (BvD), la cual se toma el periodo comprendido entre los años 2010 al 2014. Los análisis se realizan por medio de la herramienta SDATA.

\section{LA MUESTRA Y LOS DATOS}

Con el objetivo de estudiar empíricamente las hipótesis planteadas, se emplearon datos extraídos de la base de datos SABI de Bureau van Dijk $(B v D)^{3}$. Como ya se mencionó, el estudio se centró en las empresas dedicadas a la fabricación del aceite de oliva, cuyo CNAE es el 1043, du-

2. El aceite de oliva es un producto imperecedero (COOSUR, 2017), pese a que tenga una fecha de consumo preferente normalmente fijada en los 24 meses (Arribera, 2017).

3. Esta base de datos dispone de información procedente de más de 2 000000 empresas españolas y 500000 empresas portuguesas y está referida, entre otras cuestiones, a información financiera, marcas, indicadores de fortaleza financiera, directorios y contactos, declaraciones originales, estructuras corporativas detalladas, estudios de mercado, noticias de negocio y empresas, acuerdos y rumores de MyA, mapas y análisis cartográficos e informes de auditoría (Bureau van Dijk, 2017). 


\section{TABLA 1}

PRINCIPALES RESULTADOS DE LOS ESTUDIOS EMPÍRICOS

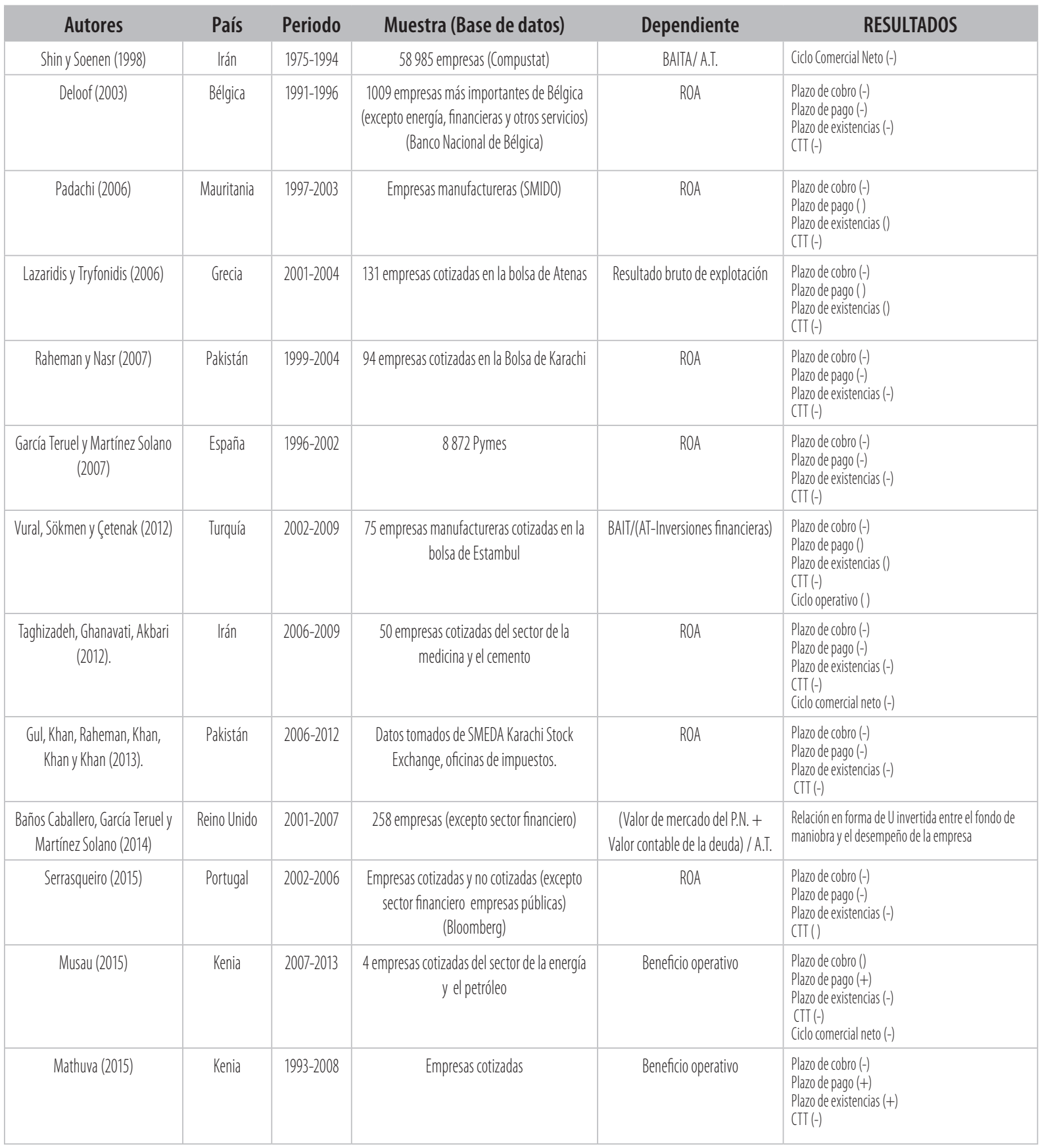

Fuente: Basado en la revisión del estado del arte.

Notas: BAIT (Beneficio antes de intereses e impuestos). BAlTA (Beneficio antes de intereses e impuestos y amortización. A.T. (Activo Total). ROA (BAIT/A.T.). P.N. (Patrimonio Neto). CTT (Ciclo de Transformación de Tesorería). (+) (-) () denotan, respectivamente, una relación positiva, negativa y no significativa entre la variable explicativa y la variable dependiente. 
rante el período que abarca desde el año 2010 hasta el 2014, ambos incluidos. Tras una primera búsqueda en $\mathrm{SABI}$, se construyó una base de datos integrada por una muestra inicial de 817 empresas. Posteriormente, se procedió a la depuración de base de datos aplicando dos filtros.

El primer filtro exigía disponer de la variable dependiente, que mediría la rentabilidad, durante tres años seguidos, con el objetivo de tener una muestra de empresas con cierta continuidad en sus datos, puesto que en el análisis econométrico se aplicaría la metodología de datos de panel. El segundo filtro fue que el ratio de endeudamiento, medido como el cociente de la suma del pasivo fijo y pasivo corriente entre el activo total, no fuese superior a 0.99, de modo que se evitó la presencia de empresas con un patrimonio negativo o igual a 0 . Tras todos estos filtros, la muestra resultante quedó integrada por un panel desbalanceado de 324 empresas.

\section{DEFINICIÓN DE LAS VARIABLES}

Al igual que Deloof (2003), Serrasqueiro (2015), García-Teruel y Martínez-Solano (2007), Padachi (2006), Jayarathne (2006) y Nazir y Afza (2009), la variable dependiente estudiada será la rentabilidad medida como BAIT/Activo Total.

Con base en la literatura previa, se seleccionaron como variables independientes aquellas más representativas de la gestión del circulante, así como otras variables de control consideradas en los estudios previos (tabla 2). Dentro del primer grupo, se encuentran: el plazo de cobro a deudores, el plazo de pago a proveedores, el plazo de almacenamiento de existencias y el CTT.

Las dos primeras variables se extrajeron directamente de SABI y, para testar su fiabilidad, se calcularon manualmente a partir de la información recogida en los estados financieros. Dado que los resultados calculados manualmente

\section{TABLA 2}

DEFINICIÓN DE LAS VARIABLES

\begin{tabular}{|c|c|c|}
\hline \multicolumn{2}{|r|}{ Variables } & Definición \\
\hline Dependiente & ROA (roa) & EBIT/Activo total \\
\hline \multirow{4}{*}{$\begin{array}{l}\text { Variables de gestión de } \\
\text { circulante }\end{array}$} & Plazo de cobro (pcobro) & $S A B \mid$ \\
\hline & Plazo de pago (pcredito) & $S A B \mid$ \\
\hline & Plazo de existencias (pexistencias) & Existencias/ (Consumo de mercaderías / 365) \\
\hline & Ciclo de tesorería (ctt) & P. cobro + P. existencias - P. pago \\
\hline \multirow{8}{*}{ Variables de control } & Endeudamiento (endeudamiento) & (Pasivo corriente + Pasivo fijo) / Activo total \\
\hline & Tamaño (tamaño) & Ln(Ventas) \\
\hline & Crecimiento (crecimiento) & (Ventas $\mathrm{t}$ - Ventas t-1) / Ventas t-1 \\
\hline & Liquidez (ac_pc) & Activo corriente/ Pasivo corriente \\
\hline & Importador (importadora) & 1 si la empresa ha realizado importaciones, 0 en caso contrario \\
\hline & Exportador (exportadora) & 1 si la empresa ha realizado exportaciones, 0 en caso contrario \\
\hline & Importador y exportador (impexp) & 1 si la empresa ha realizado importaciones y exportaciones, 0 en caso contrario \\
\hline & Cooperativas (cooperativa) & 1 si la empresa es una cooperativa, 0 en caso contrario \\
\hline
\end{tabular}

Fuente: Elaboración propia basado en las variables utilizadas para esta investigación. 
coincidieron en gran medida con aquellos extraídos de $\mathrm{SAB}$, se optó por utilizar los primeros. Por su parte, el plazo de existencias se calculó manualmente, ya que SABI no proporciona esta información ${ }^{4}$. Por último, el CTT se calculó como la suma del plazo de existencias y el plazo de cobro a clientes menos el plazo de pago a proveedores (Ciclo de tesorería $=$ P. almacenamiento + P. cobro - P. pago).

En cuanto a las variables de control se incluyeron; el endeudamiento, igual que lo emplearon Padachi (2006) o Shin y Soenen (1998); el tamaño, igual que Jayarathne (2014) y Nazir y Afza (2009); el crecimiento, como Serrasqueiro (2015) y Baños-Caballero et al. (2014); la liquidez, también empleada por Shin y Soenen (1998); una variable dicotómica que indicaba si las empresas estaban constituidas jurídicamente como cooperativas, y tres variables dicotómicas que expresaban si las empresas habían realizado importaciones, exportaciones o ambas actividades. En el caso de las variables dicotómicas, solo se disponía de datos referentes a su último año de actividad, por lo que se tomó como representativo del resto de años.

\section{ESPECIFICACIÓN DEL MODELO}

La metodología empleada ha sido la de datos de panel. La ventaja de esta técnica es que permite incorporar, además de los factores explicativos, la existencia de factores no observables que pudieran estar afectando a la rentabilidad, asociados a cada una de las empresas individualmente (ai). En concreto se estimaron modelos lineales de acuerdo con la siguiente especificación:

4. Para ello, primero se estimó la partida "Consumo de mercadería"a partir de las cifras correspondientes a "Acreedores comerciales" $y$ "Plazo de pago" que figuraban en SABI y despejando de la siguiente fórmula: Plazo de pago = Acreedores comerciales $/$ (Consumo de mercaderías / 365). Una vez despejada esta partida, se procedió a calcular el plazo de existencias siguiendo la fórmula Plazo de existencias = Existencias / (Consumo de mercaderías / 365). La cifra de "Existencias" también aparecía en SABI.

$$
\mathrm{ROA}_{i t}=\mu+X_{i t}+C_{i t}+a_{i}+\lambda_{\mathrm{t}}+_{\varepsilon i \mathrm{t}}
$$

En la ecuación anterior, el término de error $\varepsilon_{\text {it }}$ está distribuido normalmente con media 0 y varianza $\sigma_{\varepsilon}^{2}$. Por su parte, $X_{i t}$ y $C_{i t}$ denotan el conjunto de variables independientes relativas a la gestión del circulante y variables de control, respectivamente. La variable a se conoce como efecto o heterogeneidad no observada y pretende recoger las características propias de cada empresa. Dicha variable se mantiene constante en el tiempo.

Por su parte, los parámetros $\lambda_{t}$ recogen efectos temporales no observables que cambian a lo largo del tiempo, pero que son iguales para todas las empresas en cada uno de los periodos considerados. De este modo, se pretende recoger variables macroeconómicas que se escapan al control de las empresas (tipos de interés, impuestos, etc.), pero que pueden influir en su rentabilidad.

Los modelos se han estimado teniendo en cuenta tanto la posibilidad de que los efectos individuales $\left(a_{i}\right)$ estén correlacionados con las variables independientes (estimador de efectos fijos), como la posibilidad de que sean independientes de las variables explicativas (estimador de efectos aleatorios) $)^{5}$. Para elegir entre una estimación u otra, se utilizó el test de Hausman (1978).

\section{RESULTADOS EMPÍRICOS}

\section{ANÁLISIS DESCRIPTIVO}

Este apartado se destina al análisis descriptivo de las variables mencionadas. En concreto, el ROA promedio de la muestra fue positivo si bien muy reducido para todos los años estudiados (Figura 1). Además, la evolución de la rentabilidad de los activos fue creciente, si bien destaca el año 2012 por tener el menor valor de todo el periodo analizado.

5. En el segundo caso, se estima en niveles por mínimos cuadrados generalizados. 
FIGURA 1

ROA Y PLAZOS DE EXPLOTACIÓN MEDIOS (2010-2014)

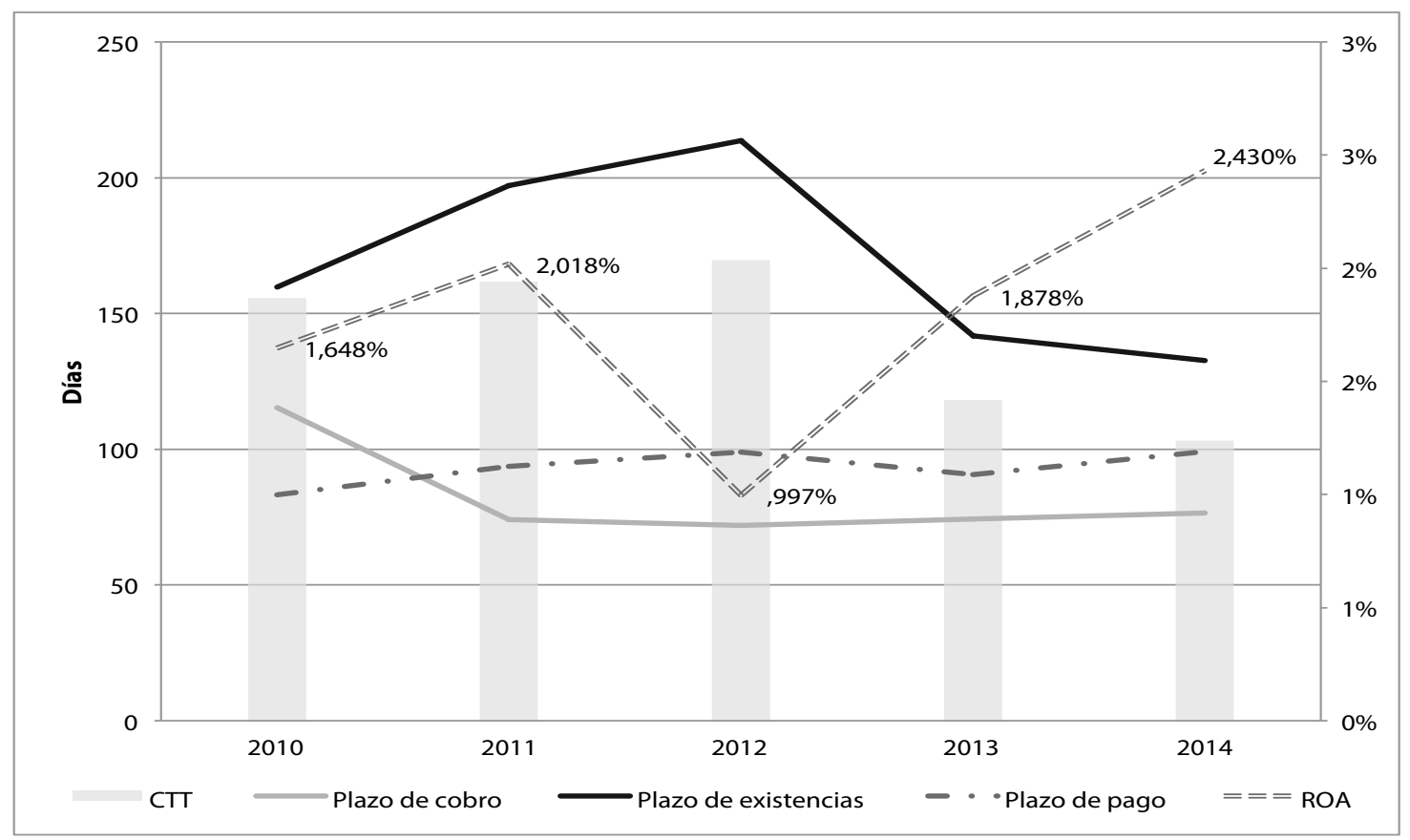

Fuente: Elaboración propia basado en el análisis de la base de datos empleada.

El plazo de cobro a clientes, con excepción de 2010, se situó en torno a los 75 días y, además, tuvo bastante estabilidad durante el periodo analizado6. Por su parte, el plazo de pago, con excepción de 2010, se sitúa por encima de los 90 días, llegando a alcanzar casi los 100 días en 2012 y 2014. Tomando los datos promedio de principio y final de periodo, se puede pensar que el plazo de crédito evolucionó favorablemente para las empresas, permitiéndoles pagar 16 días más tarde en 2014 que en 2010. Ahora bien, si este retraso en los pagos no ha sido negociado, puede estar reflejando un problema de las empresas del sector para pagar a sus proveedores en plazo.

6. El dato promedio para 2010 llama la atención, ya que supone en torno a un mes más de periodo de cobro, por lo que habría que disponer de más información para conocer las causas de dicha desviación.
El período de existencias es la de mayor magnitud de los tres plazos analizados, superando todos los años a los dos plazos anteriores. Además, aumentó entre 2010 y 2012, cayendo considerablemente los años posteriores, de modo que en 2014 el plazo de almacenamiento medio era 27 días inferior al del año 2010. Como se esperaba, dicho valor medio en los años 2011 y 2012 fue notablemente superior al del resto de años del periodo considerado, probablemente justificado por las ayudas al almacenamiento privado que estuvieron vigentes en ambos años.

Por último, podemos observar el efecto agregado de estas variables con el CTT. El tiempo medio que pasa desde que las empresas del sector pagan a sus proveedores hasta que cobran experimentó una evolución similar al plazo de existencias, incrementándose hasta 2012, año en que se situó en torno a los 170 días, y disminuyendo posteriormente hasta 103 días en 2014. 
En lo que respecta a las variables de control, el endeudamiento empresarial del sector apenas sufrió oscilaciones, pasando de un 68.8\% de deuda sobre activo total en 2010 a un $66.7 \%$ en 2014. En términos medios, dos tercios de la financiación procedían de recursos ajenos, mientras que el tercio restante consistía en recursos propios.

El crecimiento, por su parte, se midió con base en las ventas. Puede apreciarse cómo se intercalaron los años de crecimiento y decrecimiento, destacando los años 2013 y 2014, por ser el primero el año con mayor crecimiento mientras que el segundo fue el año con mayor decrecimiento.

La liquidez, medida como el ratio de activo corriente entre pasivo corriente, tuvo cierta estabilidad, durante todo el período analizado. En términos medios, las empresas del sector cuentan con 1.2 euros en activo corriente por cada euro de pasivo corriente, lo cual indica que trabajan con fondos de maniobra positivos. Dicho de otro modo, una parte de su financiación fija (propia o ajena) se destina a cubrir los activos corrientes de las empresas.

Por lo que respecta al tamaño, el volumen medio de ventas anual se situó entre los 11 y los 15 millones de euros durante todo el período. Además, aunque esta forma de medir el tamaño no se consideró en el análisis econométrico, en 2010 el número medio de empleados rondaba los 23 para posteriormente caer hasta aproximadamente los 17 trabajadores. En todo caso, la mayoría de empresas de nuestra muestra son de pequeño tamaño.

Considerando su apertura al comercio internacional, el porcentaje de las empresas exportadoras (40.4\%) superó al de las empresas importadores (7\%), algo que era de esperar visto el peso de España en la producción mundial y en las exportaciones de aceite de oliva. Por otro lado, si examinamos aquellas que realizaron ambas actividades, el porcentaje cae hasta el 6.2\% aproximadamente. Por último, las cooperativas representaban el $21 \%$ de nuestra muestra.

TABLA 3

MEDIAS DE LAS VARIABLES DE CONTROL (POR AÑO)

\begin{tabular}{|c|c|c|c|c|c|c|}
\hline & & Endeudamiento & Crecimiento & Liquidez & Ventas (miles de euros) & Número de empleados \\
\hline \multirow{2}{*}{2010} & Media & 0.69 & 0.00 & 1.21 & 11459.44 & 22.54 \\
\hline & Observaciones & 296 & 296 & 296 & 292 & 249 \\
\hline \multirow{2}{*}{2011} & Media & 0.69 & 0.07 & 1.27 & 12288.54 & 17.59 \\
\hline & Observaciones & 276 & 276 & 276 & 276 & 240 \\
\hline \multirow{2}{*}{2012} & Media & 0.67 & -0.043 & 1.23 & 11752.11 & 18.52 \\
\hline & Observaciones & 288 & 288 & 288 & 288 & 244 \\
\hline \multirow{2}{*}{2013} & Media & 0.66 & 0.23 & 1.29 & 14444.84 & 17.87 \\
\hline & Observaciones & 314 & 314 & 314 & 314 & 275 \\
\hline \multirow{2}{*}{2014} & Media & 0.67 & -0.12 & 1.29 & 12760.39 & 17.09 \\
\hline & Observaciones & 324 & 324 & 324 & 324 & 279 \\
\hline
\end{tabular}

Fuente: Resultado de la corrida de las variables estudiadas. 


\section{ANÁLISIS MULTIVARIANTE}

Para testar las hipótesis planteadas se aplicaron modelos lineales de datos de panel, tanto modelos de efectos fijos como modelos de efectos aleatorios. No obstante, el test de Hausman (1978) confirmó que el modelo de efectos fijos era más adecuado. Además, se plantearon cuatro modelos (tabla 4) que se reestimaron añadiendo el ratio del activo corriente y el pasivo corriente (tabla 5), con el objetivo de controlar la liquidez de la empresa, sin que los resultados se viesen modificados.

Los resultados empíricos obtenidos contradicen las hipótesis planteadas en el trabajo (ver tabla 6). En primer lugar, se esperaba una relación negativa entre el plazo de cobro y la rentabilidad (Hipótesis 1). Curiosamente, los resultados demostraron lo contrario y son opuestos a los obtenidos por la mayoría de trabajos empíricos.

Estos resultados podrían obedecer a varios motivos. En primer lugar, en el contexto actual de bajos tipos de interés en la economía española, el coste de oportunidad que por este motivo afrontan las empresas al prolongar los plazos de cobro es prácticamente inexistente. Más aún, las empresas del sector agrario cuya producción anual depende del abastecimiento en base a una única cosecha, pueden modular su producción, de modo que no soportan mayores costes al alargar su plazo de cobro a clientes.

Si, además, esta prolongación del plazo de cobro posibilita un aumento de las ventas (Baños-Caballero et al., 2014), estaría repercutiendo positivamente en la rentabilidad de la empresa. Así, en la medida en que buena parte de las ventas se puede estar produciendo a grandes empresas de distribución que, gracias a su poder de negociación (Briega, 2014), trabajan con plazos de pago a proveedores muy alargados, el hecho de tener un plazo de cobro elevado puede ser un indicio de trabajar con este tipo de clientes que suelen tardar en pagar pero son relativamente más segu- ros. Dicho de otra forma, puede ser un indicio de que las empresas están dando salida a su producción vía clientes pagadores.

En segundo lugar, los resultados muestran, al contrario de lo planteado en la Hipótesis 2, que a mayor plazo de almacenamiento, mayor rentabilidad. Lo anterior coincide únicamente con los resultados de Mathuva (2015). Esta relación podría estar justificada por varios motivos. Por un lado, al tratarse de un producto no perecedero y no generar unos costes de almacenamiento elevados, las empresas pueden almacenarlo para venderlo en momentos en los que los precios del producto le favorezcan, es decir, en aquellos años de menor producción de aceituna. Por otro lado, las empresas que hayan tenido un mayor periodo de almacenamiento se pueden haber beneficiado de las ayudas públicas concedidas a través de las medidas de almacenamiento privado.

En tercer lugar, al estudiar el plazo de crédito, los resultados refutan la hipótesis propuesta (Hipótesis 3), según la cual se esperaba una relación positiva con la rentabilidad, pues el hecho de que los proveedores permitan pagar más tarde a las empresas se traduciría en menores necesidades de financiación. En cambio, se observó que, al incrementarse este plazo, caía la rentabilidad de las empresas. Estos resultados coinciden con los obtenidos mayoritariamente por los trabajos empíricos (Deloof, 2003; García-Teruel y Martínez-Solano, 2007; Gul et al., 2013; Reheman y Nasr, 2007; Serrasqueiro, 2015; Taghizadeh et al., 2012). Su explicación podría ser que las empresas más rentables pagan antes, como dijo Deloof (2003), o bien que acceder a los descuentos comerciales resultase más rentable que aplazar el pago a proveedores, por ser la TAE del descuento comercial superior a la TAE que habría que pagar al solicitar fondos a una entidad de crédito, de modo que se obtuviera un diferencial positivo en forma de ahorro. 
TABLA 4

DETERMINANTES DE LA RENTABILIDAD: ESTIMACIONES

\begin{tabular}{|c|c|c|c|c|c|c|c|c|}
\hline & \multicolumn{2}{|c|}{ Modelo 1} & \multicolumn{2}{|c|}{ Modelo 2} & \multicolumn{2}{|c|}{ Model0 3} & \multicolumn{2}{|c|}{ Modelo 4} \\
\hline & E.F. & E.A. & E.F. & E.A. & E.F. & E.A. & E.F. & E.A. \\
\hline pcredito & $\begin{array}{c}-0.000^{* * *} \\
(0.000)\end{array}$ & $\begin{array}{c}-0.000^{* * *} \\
(0.000)\end{array}$ & & & & & & \\
\hline pcobro & & & $\begin{array}{l}0.000^{*} \\
(0.000)\end{array}$ & $\begin{array}{l}-0.000 \\
(0.000)\end{array}$ & & & & \\
\hline pexistencias & & & & & $\begin{array}{l}0.000^{* *} \\
(0.000)\end{array}$ & $\begin{array}{c}-0.000^{* * *} \\
(0.000)\end{array}$ & & \\
\hline $\mathrm{ctt}$ & & & & & & & $\begin{array}{c}0.000^{* *} \\
(0.000)\end{array}$ & $\begin{array}{c}-0.000 * * * \\
(0.000)\end{array}$ \\
\hline tamaño & $\begin{array}{c}0.023^{* * *} \\
(0.007)\end{array}$ & $\begin{array}{c}0.007^{* * *} \\
(0.001)\end{array}$ & $\begin{array}{c}0.029^{* * *} \\
(0.006)\end{array}$ & $\begin{array}{c}0.008^{* * *} \\
(0.001)\end{array}$ & $\begin{array}{c}0.037^{* * *} \\
(0.007)\end{array}$ & $\begin{array}{c}0.007^{* * *} \\
(0.001)\end{array}$ & $\begin{array}{c}0.033^{* * *} \\
(0.007)\end{array}$ & $\begin{array}{c}0.007^{* * *} \\
(0.001)\end{array}$ \\
\hline endeudamiento & $\begin{array}{c}-0.189^{* * *} \\
(0.024)\end{array}$ & $\begin{array}{c}-0.037^{* * *} \\
(0.001)\end{array}$ & $\begin{array}{c}-0.190^{* * *} \\
(0.023)\end{array}$ & $\begin{array}{c}-0.041^{* * *} \\
(0.010)\end{array}$ & $\begin{array}{c}-0.189^{* * *} \\
(0.026)\end{array}$ & $\begin{array}{c}-0.035^{* * *} \\
(0.010)\end{array}$ & $\begin{array}{c}-0.184^{* * *} \\
(0.026)\end{array}$ & $\begin{array}{c}-0.038^{* * *} \\
(0.010)\end{array}$ \\
\hline crecimiento & $\begin{array}{l}0.002 \\
(0.004)\end{array}$ & $\begin{array}{c}0.008^{* *} \\
(0.003)\end{array}$ & $\begin{array}{c}0.003 \\
(0.004)\end{array}$ & $\begin{array}{c}0.010^{* * *} \\
(0.003)\end{array}$ & $\begin{array}{l}0.003 \\
(0.005)\end{array}$ & $\begin{array}{c}0.010^{* *} \\
(0.003)\end{array}$ & $\begin{array}{c}0.003 \\
(0.005)\end{array}$ & $\begin{array}{c}0.011^{* * *} \\
(0.003)\end{array}$ \\
\hline importadora & $\begin{array}{l}-0.001 \\
(0.014)\end{array}$ & $\begin{array}{l}-0.005 \\
(0.007)\end{array}$ & $\begin{array}{l}-0.009 \\
(0.014)\end{array}$ & $\begin{array}{l}-0.004 \\
(0.006)\end{array}$ & $\begin{array}{l}-0.004 \\
(0.018)\end{array}$ & $\begin{array}{l}-0.004 \\
(0.006)\end{array}$ & $\begin{array}{l}-0.004 \\
(0.018)\end{array}$ & $\begin{array}{l}-0.004 \\
(0.006)\end{array}$ \\
\hline exportadora & $\begin{array}{l}-0.001 \\
(0.003)\end{array}$ & $\begin{array}{l}0.000 \\
(0.001)\end{array}$ & $\begin{array}{l}-0.002 \\
(0.003)\end{array}$ & $\begin{array}{l}-0.001 \\
(0.001)\end{array}$ & $\begin{array}{l}-0.003 \\
(0.003)\end{array}$ & $\begin{array}{l}-0.000 \\
(0.001)\end{array}$ & $\begin{array}{l}-0.003 \\
(0.003)\end{array}$ & $\begin{array}{l}-0.000 \\
(0.001)\end{array}$ \\
\hline impexp & $\begin{array}{c}0.013 \\
(0.015)\end{array}$ & $\begin{array}{l}0.006 \\
(0.007)\end{array}$ & $\begin{array}{c}0.013 \\
(0.015)\end{array}$ & $\begin{array}{l}0.006 \\
(0.007)\end{array}$ & $\begin{array}{l}0.007 \\
(0.019)\end{array}$ & $\begin{array}{l}0.006 \\
(0.007)\end{array}$ & $\begin{array}{l}0.007 \\
(0.019)\end{array}$ & $\begin{array}{l}0.006 \\
(0.007)\end{array}$ \\
\hline cooperativ & $\begin{array}{c}0.005 \\
(0.004)\end{array}$ & $\begin{array}{c}-0.021^{* * *} \\
(0.006)\end{array}$ & $\begin{array}{c}0.005 \\
(0.004)\end{array}$ & $\begin{array}{c}-0.021^{* * *} \\
(0.006)\end{array}$ & $\begin{array}{c}0.005 \\
(0.004)\end{array}$ & $\begin{array}{c}-0.021^{* * *} \\
(0.006)\end{array}$ & $\begin{array}{c}0.005 \\
(0.004)\end{array}$ & $\begin{array}{c}-0.020^{* * * *} \\
(0.006)\end{array}$ \\
\hline yr2010c & $\begin{array}{l}0.000 \\
(.)\end{array}$ & $\begin{array}{l}-0.006 \\
(0.006)\end{array}$ & $\begin{array}{l}-0.000 \\
(0.007)\end{array}$ & $\begin{array}{l}-0.006 \\
(0.006)\end{array}$ & $\begin{array}{l}-0.003 \\
(0.008)\end{array}$ & $\begin{array}{l}-0.005 \\
(0.006)\end{array}$ & $\begin{array}{l}-0.004 \\
(0.008)\end{array}$ & $\begin{array}{l}-0.005 \\
(0.006)\end{array}$ \\
\hline yr2011c & $\begin{array}{c}0.003 \\
(0.005)\end{array}$ & $\begin{array}{l}-0.003 \\
(0.006)\end{array}$ & $\begin{array}{l}0.003 \\
(0.007)\end{array}$ & $\begin{array}{l}-0.005 \\
(0.005)\end{array}$ & $\begin{array}{l}0.002 \\
(0.007)\end{array}$ & $\begin{array}{l}-0.001 \\
(0.006)\end{array}$ & $\begin{array}{c}0.001 \\
(0.007)\end{array}$ & $\begin{array}{l}-0.001 \\
(0.006)\end{array}$ \\
\hline yr2012c & $\begin{array}{l}-0.008 \\
(0.006)\end{array}$ & $\begin{array}{l}-0.013^{*} \\
(0.005)\end{array}$ & $\begin{array}{l}-0.009 \\
(0.006)\end{array}$ & $\begin{array}{c}-0.015^{* *} \\
(0.005)\end{array}$ & $\begin{array}{l}-0.009 \\
(0.006)\end{array}$ & $\begin{array}{l}-0.014^{*} \\
(0.006)\end{array}$ & $\begin{array}{l}-0.010 \\
(0.006)\end{array}$ & $\begin{array}{l}-0.014^{*} \\
(0.006)\end{array}$ \\
\hline yr2013c & $\begin{array}{l}-0.005 \\
(0.007)\end{array}$ & $\begin{array}{l}-0.005 \\
(0.005)\end{array}$ & $\begin{array}{l}-0.005 \\
(0.005)\end{array}$ & $\begin{array}{l}-0.006 \\
(0.005)\end{array}$ & $\begin{array}{l}-0.005 \\
(0.006)\end{array}$ & $\begin{array}{l}-0.006 \\
(0.005)\end{array}$ & $\begin{array}{l}-0.006 \\
(0.006)\end{array}$ & $\begin{array}{l}-0.006 \\
(0.005)\end{array}$ \\
\hline yr2014c & $\begin{array}{l}-0.001 \\
(0.008)\end{array}$ & $\begin{array}{l}0.000 \\
\text { (.) }\end{array}$ & $\begin{array}{l}0.000 \\
\text { (.) }\end{array}$ & $\begin{array}{l}0.000 \\
\text { (.) }\end{array}$ & $\begin{array}{l}0.000 \\
\text { (.) }\end{array}$ & $\begin{array}{l}0.000 \\
\text { (.) }\end{array}$ & $\begin{array}{l}0.000 \\
\text { (.) }\end{array}$ & $\begin{array}{l}0.000 \\
\text { (.) }\end{array}$ \\
\hline _cons & $\begin{array}{r}-0.019 \\
(0.053)\end{array}$ & $\begin{array}{l}0.005 \\
(0.012)\end{array}$ & $\begin{array}{l}-0.071 \\
(0.050)\end{array}$ & $\begin{array}{l}-0.001 \\
(0.012)\end{array}$ & $\begin{array}{l}-0.132^{*} \\
(0.059)\end{array}$ & $\begin{array}{c}0.004 \\
(0.012)\end{array}$ & $\begin{array}{l}-0.100^{a} \\
(0.057)\end{array}$ & $\begin{array}{c}0.004 \\
(0.012)\end{array}$ \\
\hline Observaciones & 1493 & 1493 & 1492 & 1492 & 1321 & 1321 & 1321 & 1321 \\
\hline T. de - Hausman' & & $\begin{array}{c}51.67 \\
(0.000)\end{array}$ & & $\begin{array}{c}61.22 \\
(0.000)\end{array}$ & & $\begin{array}{c}68.54 \\
(0.000)\end{array}$ & & $\begin{array}{c}66.66 \\
(0.000)\end{array}$ \\
\hline
\end{tabular}

Fuente: Resultados de la corrida en Sdata

Notas: ${ }^{* *} p=.001,{ }^{* *} p=0.01,{ }^{*} p=0.05,{ }^{a} p=0.1$. Estadístico tentre paréntesis. E.A. Efectos aleatorios, E.F. Efectos fijos. Estadísticos $X 2$ y $p$-valor del test de Hausman: contraste de igualdad entre los modelos de efectos fijos y aleatorios. Si se rechaza la hipótesis nula, sólo la estimación intragrupos (efectos fijos) resulta consistente.

Estimación consistente (modelo seleccionado). 
TABLA 5

DETERMINANTES DE LA RENTABILIDAD: ESTIMACIONES CONTROLANDO LA LIQUIDEZ

\begin{tabular}{|c|c|c|c|c|c|c|c|c|}
\hline & \multicolumn{2}{|c|}{ Modelo 1bis } & \multicolumn{2}{|c|}{ Modelo 2 bis } & \multicolumn{2}{|c|}{ Modelo 3 bis } & \multicolumn{2}{|c|}{ Modelo 4 bis } \\
\hline & E.F. & E.A. & E.F. & E.A. & E.F. & E.A. & E.F. & E.A. \\
\hline pcredito & $\begin{array}{c}-0.000^{* * *} \\
(0.000)\end{array}$ & $\begin{array}{c}-0.000^{* * *} \\
(0.000)\end{array}$ & & & & & & \\
\hline pcobro & & & $\begin{array}{l}0.000^{*} \\
(0.000)\end{array}$ & $\begin{array}{l}-0.000 \\
(0.000)\end{array}$ & & & & \\
\hline pexistencias & & & & & $\begin{array}{l}0.000^{* *} \\
(0.000)\end{array}$ & $\begin{array}{c}-0.000^{* * *} \\
(0.000)\end{array}$ & & \\
\hline $\mathrm{ctt}$ & & & & & & & $\begin{array}{l}0.000^{* *} \\
(0.000)\end{array}$ & $\begin{array}{c}-0.000^{* * *} \\
(0.000)\end{array}$ \\
\hline tamaño & $\begin{array}{c}0.023^{* * *} \\
(0.007)\end{array}$ & $\begin{array}{c}0.007^{* * *} \\
(0.001)\end{array}$ & $\begin{array}{c}0.028 * * * \\
(0.006)\end{array}$ & $\begin{array}{c}0.008^{* * *} \\
(0.001)\end{array}$ & $\begin{array}{c}0.036^{* * *} \\
(0.007)\end{array}$ & $\begin{array}{c}0.007^{* * *} \\
(0.001)\end{array}$ & $\begin{array}{c}0.033^{* * *} \\
(0.007)\end{array}$ & $\begin{array}{c}0.007^{* * *} \\
(0.001)\end{array}$ \\
\hline endeudamiento & $\begin{array}{c}-0.177^{* * *} \\
(0.025)\end{array}$ & $\begin{array}{c}-0.031^{* *} \\
(0.011)\end{array}$ & $\begin{array}{c}-0.173^{* * *} \\
(0.025)\end{array}$ & $\begin{array}{c}-0.032^{* *} \\
(0.011)\end{array}$ & $\begin{array}{c}-0.170^{* * *} \\
(0.028)\end{array}$ & $\begin{array}{l}-0.021 a \\
(0.010)\end{array}$ & $\begin{array}{c}-0.166^{* * *} \\
(0.028)\end{array}$ & $\begin{array}{l}-0.022^{*} \\
(0.011)\end{array}$ \\
\hline crecimiento & $\begin{array}{c}0.002 \\
(0.004)\end{array}$ & $\begin{array}{l}0.008^{* *} \\
(0.003)\end{array}$ & $\begin{array}{c}0.003 \\
(0.004)\end{array}$ & $\begin{array}{c}0.010^{* * *} \\
(0.003)\end{array}$ & $\begin{array}{c}0.003 \\
(0.005)\end{array}$ & $\begin{array}{c}0.011^{* * *} \\
(0.003)\end{array}$ & $\begin{array}{c}0.003 \\
(0.005)\end{array}$ & $\begin{array}{c}0.011^{* * *} \\
(0.003)\end{array}$ \\
\hline importadora & $\begin{array}{l}-0.001 \\
(0.014)\end{array}$ & $\begin{array}{l}-0.005 \\
(0.007)\end{array}$ & $\begin{array}{l}-0.009 \\
(0.014)\end{array}$ & $\begin{array}{l}-0.004 \\
(0.006)\end{array}$ & $\begin{array}{l}-0.004 \\
(0.018)\end{array}$ & $\begin{array}{l}-0.005 \\
(0.006)\end{array}$ & $\begin{array}{l}-0.003 \\
(0.018)\end{array}$ & $\begin{array}{l}-0.005 \\
(0.006)\end{array}$ \\
\hline exportadora & $\begin{array}{l}-0.001 \\
(0.003)\end{array}$ & $\begin{array}{l}0.000 \\
(0.001)\end{array}$ & $\begin{array}{l}-0.003 \\
(0.003)\end{array}$ & $\begin{array}{l}-0.001 \\
(0.001)\end{array}$ & $\begin{array}{l}-0.003 \\
(0.003)\end{array}$ & $\begin{array}{l}-0.000 \\
(0.001)\end{array}$ & $\begin{array}{l}-0.003 \\
(0.003)\end{array}$ & $\begin{array}{l}-0.000 \\
(0.001)\end{array}$ \\
\hline impexp & $\begin{array}{c}0.013 \\
(0.015)\end{array}$ & $\begin{array}{c}0.006 \\
(0.007)\end{array}$ & $\begin{array}{c}0.013 \\
(0.015)\end{array}$ & $\begin{array}{c}0.006 \\
(0.007)\end{array}$ & $\begin{array}{l}0.006 \\
(0.019)\end{array}$ & $\begin{array}{c}0.007 \\
(0.007)\end{array}$ & $\begin{array}{l}0.006 \\
(0.019)\end{array}$ & $\begin{array}{c}0.007 \\
(0.007)\end{array}$ \\
\hline cooperativ & $\begin{array}{c}0.005 \\
(0.004)\end{array}$ & $\begin{array}{c}-0.021^{* * *} \\
(0.006)\end{array}$ & $\begin{array}{c}0.005 \\
(0.004)\end{array}$ & $\begin{array}{c}-0.021^{* * *} \\
(0.006)\end{array}$ & $\begin{array}{c}0.006 \\
(0.004)\end{array}$ & $\begin{array}{c}-0.021^{* * *} \\
(0.006)\end{array}$ & $\begin{array}{c}0.006 \\
(0.004)\end{array}$ & $\begin{array}{c}-0.021^{* * *} \\
(0.006)\end{array}$ \\
\hline yr2010c & $\begin{array}{c}0.000 \\
(.)\end{array}$ & $\begin{array}{l}-0.006 \\
(0.006)\end{array}$ & $\begin{array}{c}0.000 \\
(0.007)\end{array}$ & $\begin{array}{l}-0.006 \\
(0.006)\end{array}$ & $\begin{array}{l}-0.003 \\
(0.008)\end{array}$ & $\begin{array}{l}-0.005 \\
(0.006)\end{array}$ & $\begin{array}{l}-0.004 \\
(0.008)\end{array}$ & $\begin{array}{l}-0.005 \\
(0.006)\end{array}$ \\
\hline yr2011c & $\begin{array}{c}0.003 \\
(0.005)\end{array}$ & $\begin{array}{l}-0.003 \\
(0.006)\end{array}$ & $\begin{array}{c}0.003 \\
(0.007)\end{array}$ & $\begin{array}{l}-0.005 \\
(0.005)\end{array}$ & $\begin{array}{c}0.001 \\
(0.007)\end{array}$ & $\begin{array}{l}-0.001 \\
(0.006)\end{array}$ & $\begin{array}{c}0.001 \\
(0.007)\end{array}$ & $\begin{array}{l}-0.001 \\
(0.006)\end{array}$ \\
\hline yr2012c & $\begin{array}{l}-0.008 \\
(0.006)\end{array}$ & $\begin{array}{l}-0.013^{*} \\
(0.005)\end{array}$ & $\begin{array}{l}-0.008 \\
(0.006)\end{array}$ & $\begin{array}{l}-0.015^{* *} \\
(0.005)\end{array}$ & $\begin{array}{l}-0.009 \\
(0.006)\end{array}$ & $\begin{array}{l}-0.014^{*} \\
(0.006)\end{array}$ & $\begin{array}{l}-0.009 \\
(0.006)\end{array}$ & $\begin{array}{l}-0.014^{*} \\
(0.006)\end{array}$ \\
\hline yr2013c & $\begin{array}{l}-0.005 \\
(0.007)\end{array}$ & $\begin{array}{l}-0.005 \\
(0.005)\end{array}$ & $\begin{array}{l}-0.005 \\
(0.005)\end{array}$ & $\begin{array}{l}-0.006 \\
(0.005)\end{array}$ & $\begin{array}{l}-0.005 \\
(0.006)\end{array}$ & $\begin{array}{l}-0.006 \\
(0.005)\end{array}$ & $\begin{array}{l}-0.005 \\
(0.006)\end{array}$ & $\begin{array}{l}-0.006 \\
(0.005)\end{array}$ \\
\hline yr2014c & $\begin{array}{l}-0.001 \\
(0.008)\end{array}$ & $\begin{array}{c}0.000 \\
(.)\end{array}$ & $\begin{array}{c}0.000 \\
(.)\end{array}$ & $\begin{array}{c}0.000 \\
(.)\end{array}$ & $\begin{array}{c}0.000 \\
(.)\end{array}$ & $\begin{array}{c}0.000 \\
(.)\end{array}$ & $\begin{array}{c}0.000 \\
(.)\end{array}$ & $\begin{array}{c}0.000 \\
\text { (.) }\end{array}$ \\
\hline$a c \_p c$ & $\begin{array}{c}0.007 \\
(0.005)\end{array}$ & $\begin{array}{c}0.004 \\
(0.003)\end{array}$ & $\begin{array}{l}0.010^{a} \\
(0.005)\end{array}$ & $\begin{array}{l}0.007^{*} \\
(0.003)\end{array}$ & $\begin{array}{l}0.011 a \\
(0.006)\end{array}$ & $\begin{array}{l}0.011^{* *} \\
(0.003)\end{array}$ & $\begin{array}{l}0.010^{a} \\
(0.006)\end{array}$ & $\begin{array}{l}0.011^{* * *} \\
(0.003)\end{array}$ \\
\hline _cons & $\begin{array}{l}-0.035 \\
(0.054)\end{array}$ & $\begin{array}{l}0.005 \\
(0.012)\end{array}$ & $\begin{array}{l}-0.088 \\
(0.051)\end{array}$ & $\begin{array}{l}-0.001 \\
(0.012)\end{array}$ & $\begin{array}{l}-0.154^{*} \\
(0.060)\end{array}$ & $\begin{array}{l}0.004 \\
(0.012)\end{array}$ & $\begin{array}{l}-0.121^{a} \\
(0.058)\end{array}$ & $\begin{array}{l}0.004 \\
(0.012)\end{array}$ \\
\hline Observaciones & 1493 & 1493 & 1492 & 1492 & 1321 & 1321 & 1321 & 1321 \\
\hline T. de - Hausman' & & $\begin{array}{l}52.03 \\
(0.000)\end{array}$ & & $\begin{array}{c}60.38 \\
(0.000)\end{array}$ & & $\begin{array}{l}66.07 \\
(0.000)\end{array}$ & & $\begin{array}{c}63.60 \\
(0.000)\end{array}$ \\
\hline
\end{tabular}

Fuente: Resultados de la corrida en Sdata

Notas: ${ }^{* *} \mathrm{p}=.001,{ }^{* *} \mathrm{p}=.01,{ }^{*} \mathrm{p}=.05,{ }^{\text {a }} \mathrm{p}$-valor 0. Estadístico t entre paréntesis. E.A. Efectos aleatorios, E.F. Efectos fijos. Estadísticos x2y p-valor del test de Hausman: contraste de igualdad entre los modelos de efectos fijos y aleatorios. Si se rechaza la hipótesis nula, sólo la estimación intragrupos (efectos fijos) resulta consistente.

Estimación consistente (modelo seleccionado). 
Finalmente, en lo que respecta a las variables relacionadas con el fondo de maniobra, se obtuvo una relación positiva entre el ciclo de tesorería y la rentabilidad de las empresas, contrariamente a lo planteado en la Hipótesis 4. El sentido de esta relación se podría explicar reuniendo los razonamientos empleados para las tres hipótesis anteriores, pues el ciclo de tesorería es una variable que se obtiene a partir de las tres anteriores.

\section{HALLAZGOS}

En relación con la gestión del fondo de maniobra, algunas de las variables de control utilizadas también ejercen un efecto significativo en la rentabilidad empresarial. Así, el tamaño, medido como el logaritmo de las ventas influye positivamente en la rentabilidad. Al encontrarse en un sector con márgenes operativos reducidos, el tamaño o volumen de las ventas puede ser el elemento que determine qué empresas superan el punto de equilibrio y operan con rentabilidades positivas.

También la liquidez, medida como el ratio de activo corriente entre pasivo corriente, afecta positivamente a la rentabilidad de la empresa. Este resultado coincide con los obtenidos previamente referidos a la gestión del fondo de maniobra, sugiriendo que las políticas conservadoras de ges- tión del circulante repercutirían positivamente en la rentabilidad; resultados que coinciden con los encontrados por Nazir y Afza (2009). Por el contrario, el endeudamiento de las empresas perjudica su rentabilidad económica.

\section{CONCLUSIONES}

El objeto de esta investigación ha sido analizar en qué medida la gestión del fondo de maniobra ejerce o no algún efecto sobre la rentabilidad económica de las empresas españolas dedicadas a la producción de aceite de oliva. Se construyó a partir de una base de datos integrada por una muestra inicial de 817 empresas, utilizando una muestra de 324 empresas durante el periodo 2010-2014 y tras aplicar metodología de datos de panel, se encontró que la rentabilidad, normalmente baja, de las empresas del sector se relaciona positivamente con los plazos de cobro y de existencias y negativamente con el plazo de pago a proveedores. Por lo tanto, se llega a la conclusión que la gestión del fondo de maniobra se relaciona con la rentabilidad. Lo que no está tan claro es el sentido de dicha relación.

Así, el hecho de que a mayor plazo de cobro se obtenga mayor rentabilidad puede ser, más que

TABLA 6

RESUMEN DE LAS HIPÓTESIS

\begin{tabular}{|c|c|c|}
\hline \multicolumn{1}{|c|}{ Hipótesis } & Resultado & Interpretación \\
\hline $\begin{array}{c}\text { H1: Se espera una relación negativa entre el plazo de cobro a deudores } \\
\text { y la rentabilidad de la empresa. }\end{array}$ & Se rechaza & $\begin{array}{c}\text { Resultados demostraron lo contrario y son opuestos a los } \\
\text { obtenidos por la mayoría de trabajos empíricos. }\end{array}$ \\
\hline $\begin{array}{c}\text { H2: Se espera una relación negativa entre el plazo de existencias y la } \\
\text { rentabilidad de la empresa. }\end{array}$ & Se rechaza & A mayor plazo de almacenamiento, mayor el rentabilidad. \\
\hline $\begin{array}{c}\text { H3: Se espera una relación positiva entre el plazo de pago a proveedo- } \\
\text { res y la rentabilidad de la empresa. }\end{array}$ & Se rechaza & Al incrementarse este plazo caía la rentabilidad de las empresas. \\
\hline $\begin{array}{c}\text { H4: Se espera una relación negativa entre el ciclo de tesorería y la } \\
\text { rentabilidad de la empresa. }\end{array}$ & Se rechaza & Se explica reuniendo los razonamientos empleados para las tres \\
hipótesis anteriores.
\end{tabular}

Fuente: Elaboración propia basada en los resultados del análisis multivariente. 
una relación causal, un reflejo de que las empresas con plazos de cobro más largos están vendiendo en mayor medida a compradores con gran poder de negociación, que impondrían esos plazos tan elevados. Por el contrario, la relación negativa entre el plazo de pago y la rentabilidad puede deberse a que las empresas más rentables tardan menos en pagar, como explica Deloof (2003). Finalmente, la relación positiva entre el plazo de existencias y la rentabilidad podría venir explicada en parte por las políticas de ayuda pública al almacenamiento privado aplicadas por el gobierno español en el bienio 2011-12. Los resultados anteriores llevan a recomendar a los gestores aplicar políticas conservadoras en lo que a la gestión del fondo de maniobra se refiere, ya que estas se correlacionan positivamente con la rentabilidad empresarial en el caso del sector oleícola.

Esta investigación contribuye a la literatura que analiza el papel del fondo de maniobra en la gestión empresarial de dos formas fundamentales. En primer lugar, se analiza un sector agrario, como es el sector oleícola, donde la gestión del fondo de maniobra resulta fundamental tanto por las características del tipo de producto que oferta -producción estacional, largos periodos de almacenamiento y elevada volatilidad de los precios- como por las características propias de las empresas que integran el sector-Pymes, con escaso poder de negociación con los compradores y sujetas a restricciones de acceso al crédito importante.

Tras su análisis, se comprueba que los argumentos que relacionan fondo de maniobra y rentabilidad no son generalizables al sector oleícola. Precisamente este resultado lleva a señalar como segunda contribución fundamental del estudio el haber puesto de manifiesto la necesidad de realizar un análisis más detallado de estas políticas en otros sectores con características similares al oleícola, esto es, básicamente en otros sectores agrarios.
Esta investigación no está exenta de limitaciones que abren las puertas a futuras líneas de investigación. La primera es el tamaño de la muestra estudiada, ya que representa un pequeño porcentaje del total de empresas del sector. La segunda hace referencia a las variables utilizadas para medir el carácter importador y/o exportador de las empresas, pues al tener únicamente datos para el último año de actividad, se tuvo que asumir que los datos del último año se repetían el resto de años estudiados, por lo que el estudio de su relación con la rentabilidad se vio distorsionado. Además, sería más adecuado emplear una variable cuantitativa que representase el volumen de importaciones y/o exportaciones. Por último, hubiera sido provechoso diferenciar a las almazaras envasadoras de las que únicamente vendían a granel, por lo que no se consiguió realizar un tratamiento de los datos más específico y adecuado.

\section{REFERENCIAS}

AICA. (2016). Informe de AICA sobre el mercado de aceite de oliva y el de la aceituna de mesa (campaña 2015/2016). Recuperado de http://www. upa.es/upa/_depot/_documentos/5c7d82572fa2e591459412535.pdf

Analistas económicos de Andalucía. (2012). Estudio sobre la cadena de valor en la producción y distribución del Aceite de Oliva en Andalucía. Agencia de Defensa de la Competencia de Andalucía. Recuperado de http://www.juntadeandalucia. es/defensacompetencia/sites/all/themes/competencia/files/Serie\%20estudios\%2001.pdf

Analistas económicos de Andalucía. (2013). Competencia y Cadena de Valor en la producción y distribución del Aceite de Oliva en Andalucía. Agencia de Defensa de la Competencia de Andalucía. Recuperado de http://www.infaoliva.com/documentos/documentos/Informe\%20Sectorial\%20 Aceite\%20de\%200liva.pdf

Analistas económicos de Andalucía. (2015). Informe anual del sector agrario en Andalucía 2015. Funda- 
ción Unicaja. Recuperado de https://www.unicajabanco.es/resources/fw1219756390162.pdf

Baños-Caballero, S., García-Teruel, P., y Martínez-Solano, P. (2014). Working capital management, corporate performance, and financial constraints. Journal of Business Research, 67(3), 332-338.

Briega, A. (2014). La baja rentabilidad del sector oleícola (o cómo nadie gana dinero en este sector). ¿Mito o realidad? Mercacei. Recuperado de http://www.mercacei.com/noticia/42797/ reportajes/la-baja-rentabilidad-del-sector-oleicola-o-como-nadie-gana-dinero-en-este-sector.-mito-o-realidad.html

Bureau van Dijk. (2017). Introducción. SABI. Recuperado de https://help.bvdinfo.com/mergedProjects/122_ES/Home.htm

CESCE. (2016). Informe sectorial de la economía española. Agroalimentario. Recuperado de http://www. informesectorialcesce.com/informes/3-agroalimentario/content/3-

Deloof, M. (2003). Does working capital management affect profitability of Belgian firms? Journal of business finance y Accounting, 30(3-4), 573-588.

El Economista. (2015). Las principales empresas españolas de aceite de oliva se concentran en Andalucía. Recuperado de http://www.eleconomista. es/ranking-empresas/noticias/7104712/10/15/ Las-principales-empresas-espanolas-de-aceite-de-oliva-se-concentran-en-Andalucia.html

Empresa Actual. (2010). Ranking sectorial: fabricación de aceite de oliva. Recuperado de http://www. empresaactual.com/ranking-sectorial-fabricacion-de-aceite-de-oliva/

Enow, S. T., y Brijlal, P. (2014). The effect of working capital management on profitability: The case of Small Medium and Micro Enterprises in South Africa. The Journal of Accounting and Management, 4(2).

FEGA. (2009). Anuncio de concesión de ayudas al almacenamiento privado de aceite de oliva. Ministerio de Medio Ambiente y Medio Rural y Marino. Recuperado de https://www.fega.es/sites/default/fi-
les/imported/PwfGcp/imagenes/es/AnuncioAlmacenamientoPrivadoAceite_tcm5-13347.pdf

FEGA. (2017). Ayudas al almacenamiento privado. Recuperado de https://www.fega.es/es/PwfGcp/ es/regulacion_mercados/almacenamiento_privado/index.jsp

García-Teruel, P., y Martinez-Solano, P. (2007). Effects of working capital management on SME profitability. International Journal of managerial finance, 3(2), 164-177.

Gentry, J., Vaidyanathan, R., y Lee, H. (1990). A weighted cash conversion cycle. Financial Management, 90-99.

Gul, S., Khan,M. B., Raheman, S., Khan, M. T., Khan, M., y Khan, W. (2013). Working Capital Management and Performance of SME Sector.

ICEX. (2012). Información sectorial de alimentos. Aceite de oliva. Recuperado de http://www.esenciadeolivo.es/wp-content/uploads/2013/05/informe_icex_aceite.pdf

Hausman, J. (1978). Specification Tests in Econometrics. Econometrica, 46(6), 1251-1271. doi: $10.2307 / 1913827$

Jayarathne, T. A. N. R. (2014, February). Impact of working capital management on profitability: Evidence from listed companies in Sri Lanka. In Proceedings of the 3rd International Conference on Management and Economics (Vol. 26, p. 27).

Lazaridis, I., y Tryfonidis, D. (2006). Relationship between working capital management and profitability of listed companies in the Athens stock exchange.

Llano, S. (2014). Análisis externo del sector oleícola en España.Tesis de grado. Universidad de Navarra, España.

MAGRAMA. (2015). Informe anual de la industria alimentaria española 2014-2015. Recuperado de: http:// www.mapama.gob.es/es/alimentacion/temas/ industria-agroalimentaria/_informeanualindustriaalimentaria2014-2015_tcm7-421229.pdf

MAGRAMA. (2016). Boletín del mercado de aceite de oliva. Recuperado de: http://www.mapama.gob. es/es/agricultura/temas/producciones-agrico- 
las/boletindelmercadodeaceitedeoliva-octubre2016_tcm7-442635.pdf

Mathuva, D. (2015). The Influence of working capital management components on corporate profitability. Recuperado de: https://su-plus.strathmore. edu/handle/11071/3382

Musau, N. (2015). Determinants of tax compliance among small and medium enterprises in Kenya: a case of Nairobi county. Tesis de maestría. University of Nairobi. Recuperado de http://erepository.uonbi.ac.ke/bitstream/handle/11295/95034/Musau Determinants\%20of\%20tax\%20compliance\%20 among\%20Small\%20and\%20Medium\%20Enterprises\%20in\%20Kenya.pdf?sequence=1 .

Nazir, M., y Afza, T. (2009). Impact of aggressive working capital management policy on firms' profitability. IUP Journal of Applied Finance, 15(8), 19-30.

Nobanee, H. (2009). Working capital management and firm's profitability: an optimal cash conversion cycle. Retrieved from: http://ssrn. com/abstract, 1471230.

Padachi, K. (2006). Trends in Working Capital Management and its Impact on Firms'Performance: An Analysis of Mauritian Small Manufacturing Firms. International Review of Business Research Papers, 2(2), $45-58$.

Rezaei, M., y Pourali, M. R. (2015). The Relationship between Working Capital Management Components and Profitability: Evidence from Iran. European Online Journal of Natural and Social Sciences, 4(1), 342
Raheman, A., y Nasr, M. (2007). Working Capital Management and Profitability - Case Of Pakistani Firms. International Review of Business Research Paper, 3(1), $279-300$.

Serrasqueiro, J. N. (2015). Working capital management impact on profitability: empirical study based on Portuguese firms (Doctoral dissertation).

Shin, H. H., y Soenen, L. (1998). Efficiency of working capital management and corporate profitability. Financial practice and education, 8, 37-45.

Taghizadeh, K., Ghanavati, E., Akbari, M., y Mohammadreza, E. (2012). Working Capital Management and Corporate Performance: Evidence from Iranian Companies. Procedia - Social and Behavioral Sciences, 62, 1313-1318.

Vahid, T., Elham, G., Khosroshahi Mohsen, A., y Mohammadreza, E. (2012). Working capital management and corporate performance: evidence from Iranian companies. Procedia-Social and Behavioral Sciences, 62, 1313-1318.

Vishnani, S., y Shah, B. K. (2007). Impact of working capital management policies on corporate performance-An empirical study. Global Business Review, 8(2), 267-281.

Vural, G., Sökmen, A., y Çetenak, E. (2012). Effects of working capital management on firm's performance: evidence from Turkey. International Journal of Economics and Financial Issues, 2(4), 488.

Recibido:09 de enero de 2018

Aceptado:20 de abril de 2018 